\title{
Review
}

Digestion

\section{HER2 Expression and PI3K-Akt Pathway Alterations in Gastric Cancer}

\author{
Yasutaka Sukawa $^{\text {a }}$ Hiroyuki Yamamoto $^{c}$ Katsuhiko Nosho ${ }^{a}$ Miki Ito $^{a}$ \\ Hisayoshi Igarashi ${ }^{a}$ Takafumi Naito $^{a}$ Kei Mitsuhashi ${ }^{a}$ Yasutaka Matsunaga ${ }^{a}$ \\ Taiga Takahashi $^{\mathrm{a}}$ Masashi Mikami $^{\mathrm{a}}$ Yasushi Adachi $^{\mathrm{a}}$ Hiromu Suzuki $^{\mathrm{b}}$ \\ Yasuhisa Shinomura ${ }^{a}$
}

Departments of a Gastroenterology, Rheumatology and Clinical Immunology and ${ }^{b}$ Molecular Biology, Sapporo Medical University School of Medicine, Sapporo, and ' Division of Gastroenterology and Hepatology, Department of Internal Medicine, St. Marianna University School of Medicine, Kawasaki, Japan

\section{Key Words}

HER2 - Gastric cancer · PI3K · Akt · Phosphate and tensin homolog $\cdot$ PIK3CA $\cdot$ Trastuzumab

\begin{abstract}
The anti-HER2 antibody trastuzumab has led to an era of personalized therapy in gastric cancer (GC). As a result, HER2 expression has become a major concern in GC. HER2 overexpression is seen in $7-34 \%$ of GC cases. Trastuzumab is an antibody that targets the HER2 extracellular domain and induces antibody-dependent cellular cytotoxicity and inhibition of the HER2 downstream signals. Mechanisms of resistance to trastuzumab have been reported in breast cancer. There are various mechanisms underlying trastuzumab resistance, such as alterations of HER2 structure or surroundings, dysregulation of HER2 downstream signal effectors and interaction of HER2 with other membrane receptors. The PI3K-Akt pathway is one of the main downstream signaling pathways of HER2. It is well known that PIK3CA mutations and phosphate and tensin homolog (PTEN) inactivation cause over-activation of the downstream signal without an upstream signal activation. Frequencies of PIK3CA mutations and PTEN inactivation have been reported to be 4-25 and $16-77 \%$, respectively. However, little is known about
\end{abstract}

the association between HER2 expression and PI3K-Akt pathway alterations in GC. We have found that HER2 overexpression was significantly correlated with pAkt expression in GC tissues. Furthermore, pAkt expression was correlated with poor prognosis. These results suggest that the PI3K-Akt pathway plays an important role in HER2-positive GC. Moreover, PIK3CA mutations and/or PTEN inactivation might affect the effectiveness of HER2-targeting therapy. Hence, it is necessary to clarify not only HER2 alterations but also PI3KAkt pathway alterations for HER2-targeting therapy in GC. This review will introduce recent investigations and consider the current status of HER2-targeted therapy for treatment of GC.

(c) 2014 S. Karger AG, Basel

\section{Significance of HER2 Expression in Gastric Cancer}

Gastric cancer (GC) is one of the most common cancers and the second leading cause of cancer-related death in the world [1]. Trastuzumab is the first molecular target drug in GC and it brought about an era of personalized therapy. Trastuzumab, a monoclonal antibody that targets the HER2 extracellular domain, inhibits HER2 downstream signal activation and induces antibody-dependent

\section{KARGER}

E-Mail karger@karger.com www.karger.com/dig (c) 2014 S. Karger AG, Basel

0012-2823/14/0891-0012\$39.50/0
Yasutaka Sukawa, MD, PhD

Department of Medical Oncology

Dana Farber Cancer Institute, 450 Brookline Avenue

Boston, MA 02215 (USA)

E-Mail yasutaka_sukawa@dfci.harvard.edu 
cellular cytotoxicity. Trastuzumab has shown a survival advantage in patients with breast cancer with HER2 overexpression.

In the ToGA study, standard chemotherapy regimens (capecitabine plus cisplatin or fluorouracil plus cisplatin) with trastuzumab resulted in longer survival times than those without trastuzumab in patients with HER2-positive GC [2]. Median overall survival periods were 13.8 months in patients assigned to a trastuzumab plus chemotherapy group and 11.1 months in patients assigned to a chemotherapy alone group (hazard ratio $0.74, \mathrm{p}=$ 0.0046).

In a post hoc analysis, patients were divided into two subgroups, with either a high level (immunohistochemistry, IHC, 2+ and FISH-positive or IHC 3+) or low level (IHC 0 and FISH-positive or IHC $1+$ and FISH-positive) of HER2 expression in the tumors. Median overall survival periods were 16.0 months in patients assigned to the trastuzumab plus chemotherapy group and 11.8 months in those assigned to the chemotherapy alone group [2]. Consequently, HER2-positive GC is generally defined as a tumor with IHC 2+ and FISH-positive or IHC $3+$.

In addition to the effectiveness of trastuzumab as a first-line chemotherapy, a role of trastuzumab after firstline chemotherapy has recently been receiving increasing attention. It has previously been demonstrated that paclitaxel plus trastuzumab as the second-line chemotherapy in HER2-positive GC showed a good response rate $(37 \%$ at 16 weeks) without additional severe side effects. Furthermore, many clinical trials are ongoing to evaluate the effectiveness of trastuzumab beyond progressive disease. The effectiveness of trastuzumab has already been proven in patients with breast cancer who failed with trastuzumab-combined first-line chemotherapy.

HER 2 overexpression is observed in $7-34 \%$ of GC cases [2-6] and is correlated with clinicopathological features, including depth of tumor invasion, lymph node metastasis, intestinal-like subtype and tumor stage. However, a prognostic significance of HER 2 overexpression in patients with GC is still controversial, unlike in breast cancer. Hence, HER2 expression has become a major concern in GC.

\section{Mechanisms of Resistance to Trastuzumab Therapy}

A number of studies on breast cancer have led to the identification of factors associated with inherent or treatment-acquired trastuzumab resistance (fig. 1).

\section{Alterations of HER2 Structure or Surroundings}

HER2 is a member of the epidermal growth factor receptor (EGFR) family and consists of an extracellular domain and an intracellular domain. HER2 can activate its downstream signal by dimerization, both as a homodimer and a heterodimer with other EGFR family receptors. Alterations such as increased heterodimerization of HER2 with EGFR or HER3 are thought to induce resistance to trastuzumab therapy. Some proteins, such as the membrane-associated glycoprotein MUC4, are known to mask HER2 and prevent trastuzumab from binding to HER2 [7]. Cleavage of the full-length $185-\mathrm{kDa}$ HER2 protein produces a $110-\mathrm{kDa}$ ECD and a $95-\mathrm{kDa}$ $\mathrm{NH} 2$ terminal-truncated membrane-associated fragment (p95HER2) with increased kinase activity.p95HER2 expression has been found in about $30 \%$ of breast cancers [8], and patients with p95HER2 showed poor prognosis compared to patients with full-length HER2 [9].

\section{Dysregulation of Downstream Signaling Effectors}

The phosphatidylinositol-3-kinase (PI3K) pathway is one of the most important signaling pathways in several malignancies because it activates cell proliferation, cell survival, motility and cell growth [10]. This pathway is activated by many growth factor receptors, including HER2, and transduces the signals (fig. 2). Therefore, PI3K pathway activation is associated with resistance to trastuzumab and trastuzumab exerts its antitumor effects only in the presence of a normal PI3K pathway in HER2 overexpressing breast cancer [11, 12]. A tumor suppressor gene, phosphatase and tensin homolog (PTEN) deleted on chromosome 10, negatively regulates the PI3K-Akt pathway. It is well known that dysregulation of the PI3K pathway plays an important role in the development of malignancy and the most common alterations in this pathway are PIK3CA mutations and PTEN inactivation [13, 14], both of which can lead to constitutive activation of the PI3K pathway, resulting in resistance to trastuzumab therapy [15].

Interactions of HER 2 with Other Membrane Receptors Interactions and cross-signaling between HER2 and other growth factor receptors may also cause resistance to trastuzumab. For example, insulin-like growth factor receptor 1 (IGF1R) can activate the PI3K-Akt pathway independently of the HER2 signal. Inhibition of IGF1R with an anti-receptor antibody or an IGF1R-specific tyrosine kinase inhibitor sensitizes cells that are resistant to trastuzumab [16]. Moreover, formation of HER2-IGF1R heterodimers results in phosphorylation and activation 


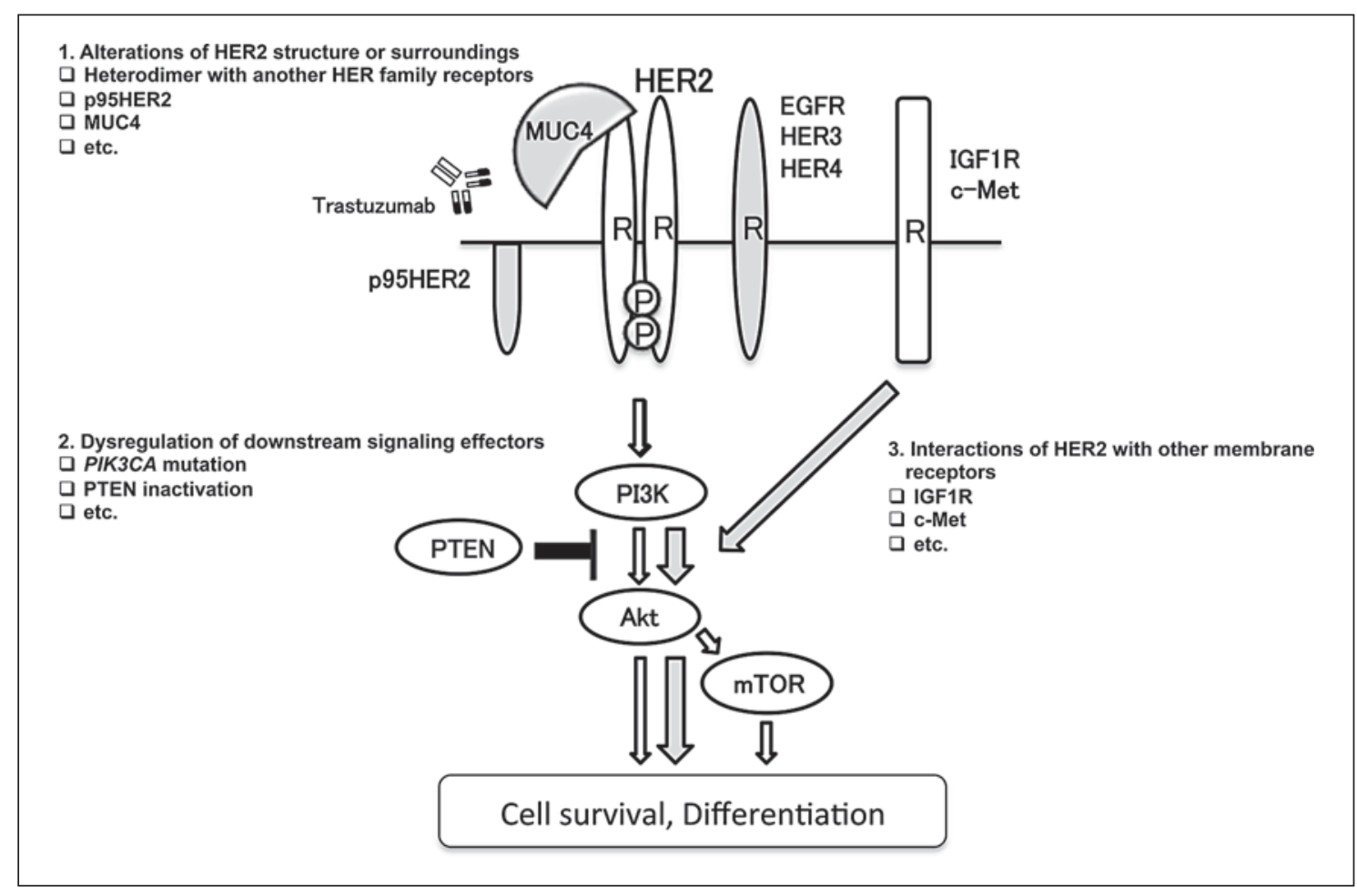

Fig. 1. Mechanisms of resistance to trastuzumab therapy. There are three major mechanisms of resistance to trastuzumab, which binds HER2 and inhibits HER2 signaling. The first mechanism is alteration of HER2 structure or surroundings. This either loses trastuzumab binding site, prevents trastuzumab from binding to HER2 or transduces HER2 signal by heterodimerization which cannot be inhibited by trastuzumab. The second mechanism is dysregulation of downstream signaling effectors such as PIK3CA mutation or PTEN inactivation. In this case, the downstream signal can be activated without upstream signals. The third is interactions of HER2 with other membrane receptors.
Fig. 2. The PI3K-Akt pathway. PI3K phosphorylates PIP2 [phosphatidylinositol $(3,4)$-bisphosphate] and produces PIP3 [phosphatidylinositol (3,4,5)-trisphosphate]. Subsequently, PIP3 activates Akt by phosphorylation of Akt and induces cell proliferation, survival, migration and differentiation. PTEN dephosphorylates PIP3 and inhibits the activation of Akt by PIP3.

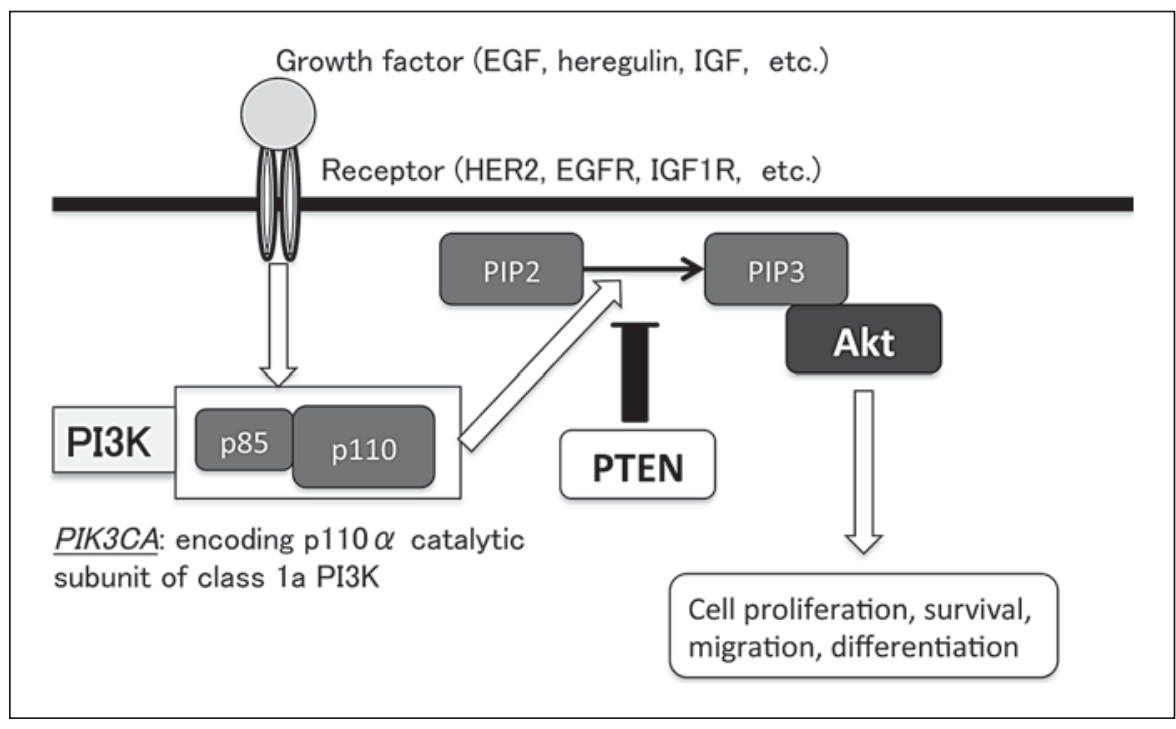


of HER2 via stimulation of IGF1R by ligands in trastuzumab-resistant breast cancer cells [17]. On the other hand, preclinical studies have shown that lapatinib is able to block the HER2 signal and overcome IGF1R signaling even in the presence of IGF1 [17]. MET, known as a hepatocyte growth factor receptor, also has some interactions with the HER2 signal and therefore might cause trastuzumab resistance [18]. Thus, further understanding of the interactions of HER2 with other membrane receptors is also important for personalized therapy in GC.

\section{Relationship between HER2 Expression and PI3K-Akt Pathway Alterations in GC}

\section{PIK3CA Mutations in GC}

$P I K 3 C A$ is mutated in a wide variety of human tumor types, including GC. Activating mutations in this gene upregulate the PI3K-AKT signaling pathway, making it a potentially useful therapeutic target. Furthermore, PIK3CA mutations have been receiving increasing attention because Liao et al. [19] showed in a large cohort study that aspirin intake prolonged survival time in patients who had colorectal cancer with PIK3CA mutations. Pyrosequencing-based methods facilitate the identification of low-frequency tumor mutations and allow more accurate assessment of the tumor mutation burden [20-22]. We determined PIK3CA mutations in GC tissues by using pyrosequencing [23]. The overall prevalence of PIK3CA mutations in that study was $8.7 \%$, a value that is within the range (4.3-15.9\%) of values in the currently available literature [24]. The mutation frequency was high (21.4\%) in T4 cancers and low (6.4\%) in T2 cancers. Thus, PIK3CA mutations appear to be late events in gastric carcinogenesis, leading to tumor progression.

The most common PIK3CA mutation was H1047R as described previously [24]. Importantly, two new types of mutations were found in exon 1 . To our knowledge, mutations involving amino acids 88 and 108 (R88Q and R108H) have never been reported before in GC, nor do they appear in the COSMIC database, despite the large number of studies in which that region was investigated. These mutations have been detected in several other types of cancer tissues [25]. Importantly, these mutations have been reported to be gain-of-function mutations [26]. The results have potential clinical implications since the mutational status of PIK3CA could stratify patients for genotype-based molecular therapies targeting the PI3K pathway. Thus, exon 1 should be analyzed in GC in those clinical settings.
In our analysis, the frequency of pAkt expression was higher in cancers with exon 20 mutations (100\%) than in those with exon 1 (40\%) or exon 9 (56\%) mutations, although the difference did not reach statistical significance. This finding is consistent with the results of a previous study showing that some PIK3CA mutations, mainly exon 9 mutations, caused Akt-independent pathway activation in a breast cancer cell line [27]. These results further support the notion that the functional significance of PIK3CA mutations depends on the mutation type and that the H1047R hotspot mutation has high oncogenic activity.

\section{PTEN Alterations in GC}

The tumor suppressor gene PTEN is located on chromosome 10q23.3 and acts as a plasma membrane lipid phosphatase. Its primary target for dephosphorylation is the second messenger phosphatidylinositol-3,4,5-triphosphate, the product of PI3K. Thereby, PTEN negatively regulates the PI3K-Akt pathway. Loss or downregulation of PTEN function due to mutations, haploinsufficiency from loss of heterozygosity and/or epigenetic down-modulation has been reported in GC. These PTEN alterations were detected in 16-77\% of GC and were associated with poor prognosis, deeper invasion, lymph node metastasis and advanced stage [28]. However, the results are conflicting because there are many ways of evaluating PTEN alterations, such as sequencing, FISH, IHC and methylation. Furthermore, we have no definite criteria for PTEN inactivation in IHC analysis. Hence, establishment of a method for evaluation of PTEN alterations and further clarification of the role of PTEN alterations in GC are now of utmost importance.

Recently, Yang et al. [29] reported that miR-21 induced cell survival and cisplatin resistance through downregulation of the expression of PTEN and activation of Akt in GC cells. PTEN may play an important role in resistance to not only HER2-targeting therapy, but also cytotoxic agents such as cisplatin.

\section{HER 2 and PI3K-Akt Pathway Alterations in GC}

We previously reported associations among HER2 expression, PIK3CA mutations and pAkt expression in GC [23]. In our analysis, HER 2 overexpression (IHC 3+) was present in 20 samples (8.4\%), a value that is within the range (7-34\%) of values in the currently available literature [2-6]. HER2 overexpression was significantly correlated with intestinal histological type. We could not find any correlation between HER 2 expression and poor prognosis, unlike that in breast cancer. Some studies have suggested that HER2 positivity in GC is associated with poor 
prognosis and aggressive disease, but the results are conflicting. It is important to consider that there is heterogeneity in HER2 expression in GC. Although HER-2-positive cancer cells were not always consistent with pAktpositive cancer cells in each tumor tissue specimen, HER2 overexpression was significantly correlated with pAkt expression in GC tissues ( 80 vs. $49 \%, \mathrm{p}<0.01$ ). Moreover, pAkt expression was correlated with poor prognosis. Thus, the HER2-Akt axis appears to play an important role in HER2-overexpressing GC.

PIK3CA mutation is one of the mechanisms underlying the resistance to trastuzumab in breast cancer. In our study, PIK3CA mutations were present in only 1 case of GC with HER2 expression of IHC 2+. However, data for the analysis consisted mainly of data for patients without indication for chemotherapy. Therefore, we should accumulate more data on patients with advanced cancer with indication for trastuzumab therapy to evaluate whether PIK3CA mutation is a major mechanism underlying the resistance to trastuzumab in GC.

To our knowledge, an association between HER2 expression and PTEN alterations in GC has not been clarified. We are investigating PTEN expression in our GC samples. It seems that PTEN inactivation occurs independently of HER2 expression. PTEN inactivation may play a more important role than PIK3CA mutations in determining the effectiveness of HER2-targeting therapy because PTEN inactivation is more frequent than PIK3CA mutations.

We previously reported that DNA methylation plays an important role in GC [30]. Since DNA methylation is one of the key mechanisms underlying PTEN inactivation, we are also trying to clarify the clinical significance of epigenetic alterations in HER2-overexpressing GC.

\section{Development of a New Therapeutic Strategy in GC}

A number of drugs targeting HER2 or its downstream signals are under development, including an ongoing phase 3 clinical trial in GC (table 1). In some clinical trials, PIK3CA mutation or PTEN loss has been evaluated as a possible predictive biomarker and has also been used as one of the inclusion criteria. This is why an understanding of the association between HER2 expression and PI3K-Akt pathway alterations is necessary to develop a new therapeutic strategy not only with trastuzumab, but also with other new molecular target drugs.

A prospective study is necessary for revealing a new biomarker for trastuzumab therapy in GC. We have two
Table 1. Drugs targeting HER2 and the PI3K-Akt pathway that are under ongoing clinical trials for gastric cancer

\begin{tabular}{lll}
\hline Target & Drug & Phase \\
\hline HER2 & T-DM1 & 3 \\
& MGAH22 & 1 \\
\hline EGFR/HER2 & Lapatinib & 3 \\
& ASLAN001 & 2 \\
& Afatinib & 2 \\
\hline HER2/HER3 & LJM716 & 1 \\
& MM111 & 2 \\
\hline pan-HER & HM781-36B & $1 / 2$ \\
\hline HER dimerization & Pertuzumab & 3 \\
\hline PI3K & BYL719 & 1 \\
\hline PI3K/Akt/mTOR & Everolimus & 3 \\
\hline Akt & MK-2206 & 2 \\
\hline
\end{tabular}

ongoing analyses. One is an exploratory analysis using serum samples in a phase 2 study to evaluate the efficacy and safety of S-1 plus cisplatin with trastuzumab therapy in HER2-positive GC (WJOG7212G; T-SPACE study). Another is an analysis using serum and cancer tissue samples in a phase 2 study to evaluate the efficacy of continuing trastuzumab beyond progressive disease for patients after failure of first-line chemotherapy with trastuzumab (WJOG7112G; T-ACT study). In these studies, we are going to analyze alterations of HER2-related factors including the PI3K-Akt pathway to identify a new predictive biomarker for trastuzumab therapy.

\section{Conclusion}

Trastuzumab has led to an era of personalized therapy in GC. The PI3K-Akt pathway is one of the critical downstream signals from HER2. PI3K-Akt pathway alterations are expected to be biomarkers for predicting the effectiveness of trastuzumab therapy. Clarification of roles of the PI3K-Akt pathway alterations may also contribute to the development of new molecular target drugs.

\section{Disclosure Statement}

The authors have no conflicts of interest to declare.
16

Digestion 2014;89:12-17 DOI: $10.1159 / 000356201$
Sukawa et al. 


\section{References}

1 Hamilton JP, Meltzer SJ: A review of the genomics of gastric cancer. Clin Gastroenterol Hepatol 2006;4:416-425.

-2 Bang YJ, Van Cutsem E, Feyereislova A, Chung HC, Shen L, Sawaki A, Lordick F, Ohtsu A, Omuro Y, Satoh T, Aprile G, Kulikov E, Hil J, Lehle M, Rüschoff J, Kang YK: Trastuzumab in combination with chemotherapy versus chemotherapy alone for treatment of HER2-positive advanced gastric or gastro-oesophageal junction cancer (ToGA): a phase 3, open-label, randomised controlled trial. Lancet 2010;376:687-697.

$\checkmark 3$ Gravalos C, Jimeno A: HER2 in gastric cancer: a new prognostic factor and a novel therapeutic target. Ann Oncol 2008;19:15231529.

4 Hofmann M, Stoss O, Shi D, Büttner R, Van de Vijver M, Kim W, Ochiai A, Rüschoff J, Henkel T: Assessment of a HER2 scoring system for gastric cancer: results from a validation study. Histopathology 2008;52:797-805.

5 Sawaki A, Ohashi Y, Omuro Y, Satoh T, Hamamoto Y, Boku N, Miyata Y, Takiuchi H, Yamaguchi K, Sasaki Y, Nishina T, Satoh A, Baba E, Tamura T, Abe T, Hatake K, Ohtsu A Efficacy of trastuzumab in Japanese patients with HER2-positive advanced gastric or gastroesophageal junction cancer: a subgroup analysis of the Trastuzumab for Gastric Cancer (ToGA) study. Gastric Cancer 2012;15: 313-322.

6 Tanner M, Hollmén M, Junttila TT, Kapanen AI, Tommola S, Soini Y, Helin H, Salo J, Joensuu H, Sihvo E, Elenius K, Isola J: Amplification of HER-2 in gastric carcinoma: association with Topoisomerase II $\alpha$ gene amplification, intestinal type, poor prognosis and sensitivity to trastuzumab. Ann Oncol 2005 16:273-278.

7 Pályi-Krekk Z, Barok M, Isola J, Tammi M, Szöllosi J, Nagy P: Hyaluronan-induced masking of ErbB2 and CD44-enhanced trastuzumab internalisation in trastuzumab resistant breast cancer. Eur J Cancer 2007;43: 2423-2433.

$>8$ Molina MA, Sáez R, Ramsey EE, GarciaBarchino MJ, Rojo F, Evans AJ, Albanell J, Keenan EJ, Lluch A, García-Conde J, Baselga J, Clinton GM: $\mathrm{NH}_{2}$-terminal truncated HER2 protein but not full-length receptor is associated with nodal metastasis in human breast cancer. Clin Cancer Res 2002;8:347-353.

$\checkmark$ Scaltriti M, Rojo F, Ocaña A, Anido J, Guzman M, Cortes J, Di Cosimo S, Matias-Guiu X, Ramon y Cajal S, Arribas J, Baselga J: Expression of $\mathrm{p} 95 \mathrm{HER} 2$, a truncated form of the HER2 receptor, and response to anti-HER2 therapies in breast cancer. J Natl Cancer Inst 2007;99:628-638.

$>10$ Engelman JA, Luo J, Cantley LC: The evolution of phosphatidylinositol 3-kinases as regulators of growth and metabolism. Nat Rev Genet 2006;7:606-619.
11 Fabi A, Metro G, Di Benedetto A, Nisticò C, Vici P, Melucci E, Antoniani B, Perracchio L, Sperduti I, Milella M, Cognetti F, Mottolese M: Clinical significance of PTEN and p-Akt co-expression in HER2-positive metastatic breast cancer patients treated with trastuzumab-based therapies. Oncology 2010;78:141149.

12 Kataoka Y, Mukohara T, Shimada H, Saijo N, Hirai M, Minami H: Association between gain-of-function mutations in PIK3CA and resistance to HER2-targeted agents in HER2amplified breast cancer cell lines. Ann Oncol 2010;21:255-262.

13 Brugge J, Hung MC, Mills GB: A new mutational AKTivation in the PI3K pathway. Cancer Cell 2007;12:104-107.

14 Coughlin CM, Johnston DS, Strahs A, Burczynski ME, Bacus S, Hill J, Feingold JM, Zacharchuk C, Berkenblit A: Approaches and limitations of phosphatidylinositol-3-kinase pathway activation status as a predictive biomarker in the clinical development of targeted therapy. Breast Cancer Res Treat 2010;124 $1-11$.

15 Berns K, Horlings HM, Hennessy BT, Madiredjo M, Hijmans EM, Beelen K, Linn SC, Gonzalez-Angulo AM, Stemke-Hale K, Hauptmann M, Beijersbergen RL, Mills GB, van de Vijver MJ, Bernards R: A functional genetic approach identifies the PI3K pathway as a major determinant of trastuzumab resistance in breast cancer. Cancer Cell 2007;12: 395-402.

16 Nahta R, Yuan LX, Zhang B, Kobayashi R, Esteva FJ: Insulin-like growth factor-I receptor/ human epidermal growth factor receptor 2 heterodimerization contributes to trastuzumab resistance of breast cancer cells. Cancer Res 2005;65:11118-11128.

17 Bender LM, Nahta R: Her2 cross talk and therapeutic resistance in breast cancer. Front Biosci 2008;13:3906-3912.

18 Shattuck DL, Miller JK, Carraway KL 3rd, Sweeney C: Met receptor contributes to trastuzumab resistance of Her2-overexpressing breast cancer cells. Cancer Res 2008;68: 1471-1477.

19 Liao X, Lochhead P, Nishihara R, Morikawa T, Kuchiba A, Yamauchi M, Imamura Y, Qian ZR, Baba Y, Shima K, Sun R, Nosho K, Meyerhardt JA, Giovannucci E, Fuchs CS, Chan AT, Ogino S: Aspirin use, tumor PIK3CA mutation, and colorectal-cancer survival. N Engl J Med 2012;367:1596-1606.

20 Baba Y, Nosho K, Shima K, Hayashi M, Meyerhardt JA, Chan AT, Giovannucci E, Fuchs CS, Ogino S: Phosphorylated AKT expression is associated with PIK3CA mutation, low stage, and favorable outcome in 717 colorectal cancers. Cancer 2011;117:1399-1408.
21 Nosho K, Kawasaki T, Ohnishi M, Suemoto Y, Kirkner GJ, Zepf D, Yan L, Longtine JA, Fuchs CS, Ogino S: PIK3CA mutation in colorectal cancer: relationship with genetic and epigenetic alterations. Neoplasia 2008; 10 : 534-541.

22 Weidlich S, Walsh K, Crowther D, Burczynski ME, Feuerstein G, Carey FA, Steele RJ, Wolf CR, Miele G, Smith G: Pyrosequencingbased methods reveal marked inter-individual differences in oncogene mutation burden in human colorectal tumours. Br J Cancer 2011;105:246-254.

23 Sukawa Y, Yamamoto H, Nosho K, Kunimoto H, Suzuki H, Adachi Y, Nakazawa M, Nobuoka T, Kawayama M, Mikami M, Matsuno T, Hasegawa T, Hirata K, Imai K, Shinomura Y: Alterations in the human epidermal growth factor receptor 2-phosphatidylinositol 3-kinase-v-Akt pathway in gastric cancer. World J Gastroenterol 2012;18:6577-6586.

24 Barbi S, Cataldo I, De Manzoni G, Bersani S, Lamba S, Mattuzzi S, Bardelli A, Scarpa A: The analysis of PIK3CA mutations in gastric carcinoma and metanalysis of literature suggest that exon-selectivity is a signature of cancer type. J Exp Clin Cancer Res 2010;29:32.

25 Rudd ML, Price JC, Fogoros S, Godwin AK, Sgroi DC, Merino MJ, Bell DW: A unique spectrum of somatic PIK3CA (p110a) mutations within primary endometrial carcinomas. Clin Cancer Res 2011;17:1331-1340.

26 Oda K, Okada J, Timmerman L, RodriguezViciana P, Stokoe D, Shoji K, Taketani Y, Kuramoto H, Knight ZA, Shokat KM, McCormick F: PIK3CA cooperates with other phosphatidylinositol $3^{\prime}$-kinase pathway mutations to effect oncogenic transformation. Cancer Res 2008;68:8127-8136.

27 Vasudevan KM, Barbie DA, Davies MA, Rabinovsky R, McNear CJ, Kim JJ, Hennessy BT, Tseng H, Pochanard P, Kim SY, Dunn IF, Schinzel AC, Sandy P, Hoersch S, Sheng Q, Gupta PB, Boehm JS, Reiling JH, Silver S, Lu Y, Stemke-Hale K, Dutta B, Joy C, Sahin AA, Gonzalez-Angulo AM, Lluch A, Rameh LE, Jacks T, Root DE, Lander ES, Mills GB, Hahn WC, Sellers WR, Garraway LA: AKT-independent signaling downstream of oncogenic PIK3CA mutations in human cancer. Cancer Cell 2009;16:21-32.

28 Mina S, Bohn BA, Simon R, Krohn A, Reeh M, Arnold D, Bokemeyer C, Sauter G, Izbicki JR, Marx A, Stahl PR: PTEN deletion is rare but often homogeneous in gastric cancer. J Clin Pathol 2012;65:693-698.

29 Yang SM, Huang C, Li XF, Yu MZ, He Y, Li J: miR-21 confers cisplatin resistance in gastric cancer cells by regulating PTEN. Toxicology 2013;306:162-168.

-30 Yamamoto E, Suzuki H, Takamaru H, Yamamoto H, Toyota M, Shinomura Y: Role of DNA methylation in the development of diffuse-type gastric cancer. Digestion 2011;83: 241-249. 